\title{
PHOTOMETRIC AND RADIAL VELOCITY VARIATIONS OF RCrB NEAR MAXIMUM LIGHT
}

\author{
A.V. Raveendran, B.N. Ashoka, N.Kameswar a Rao \\ Ind $i$ an Institute of Astrophysics \\ Banga lore 560034, India
}

ABSTRACT: Fourier analysis of the light curves of RCrB in $V$ band near maximum shows that in addition to several significant short periods there is a modulation of the visual light with a period around 1170 day, similar to that of $L$ band $f$ lux, noticed by strecker. This indicates that there is some contribution to the visual light variations of the star from the pulsating circumstellar dust. Radial ve locities of $R \quad C r B$ obtained at Kavalur during FebruaryMay 1985 show variations with a period around 47 days.

\section{INTRODUCTION}

In the $\mathrm{c}$ ase of $\mathrm{R} \mathrm{CrB}$, the light near maximum fluctuates with an amp litude in the range of about 0.1-0.3 mag and appears very irregu lar in shape, amplitude and period. In order to check the possibility that the apparent irregular behaviour is as a result of the superposition of several independent waves, we decided to Fourier analyse the avai lable light curves of $R$ CrB obtained near maximum. The technique of Discrete Fourier Transform as developed by Deeming (1975) was employed, since the information on aliasing due to the different data spacings can be obtained directly from the spectral window derived from the times of observations. All the availab le measurements in $V$ band from 1971 to 1983, except those be longing to either a primary or a secondary fading, were included in the ana lysis.

\section{POWER SPECTRUM}

Several peaks of significant and comparable amp litudes could be identified in the power spectrum. We made a least square solution for the determination of the optimum periods and their corresponding amp litudes and phases of the dominant peaks. We found that with these quantities the observed light curves could not be reproduced satisfactorily. This prompted us to suspect that the various periodicities had their origins from different data segments obtained at different epochs.

191

K. Hunger et al. (eds.), Hydrogen Deficient Stars and Related Objects, 191-195.

(C) 1986 by D. Reidel Publishing Company. 


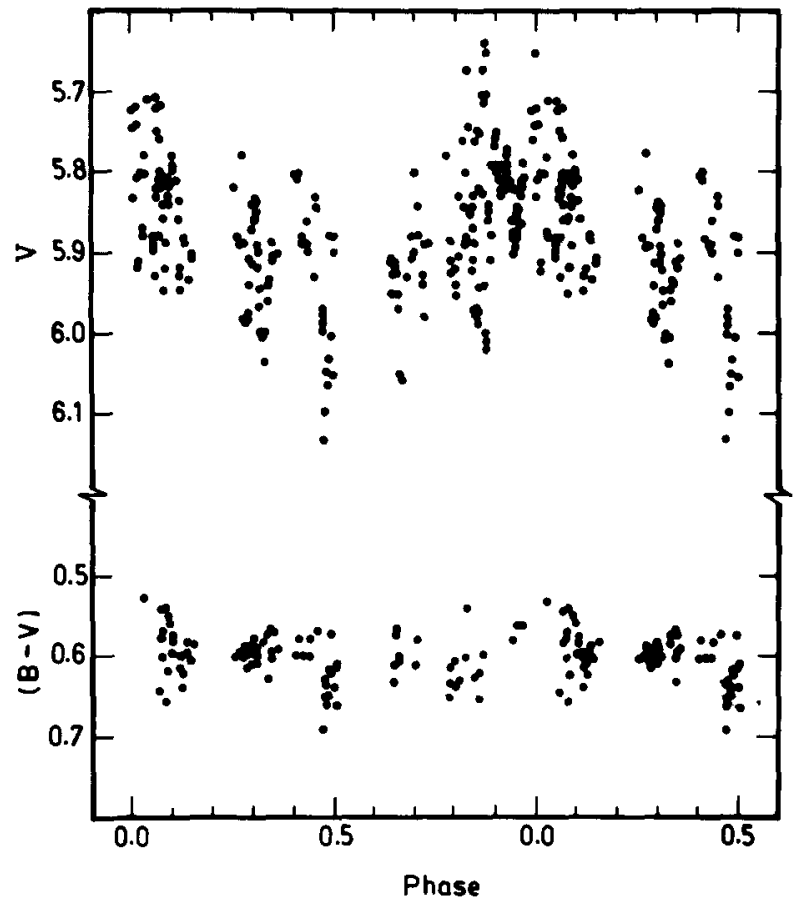

Fig. 1a. $V$ and $B-V$ values of $R C r B$ near maximum during 1971-83 folded over a 1170 day period.

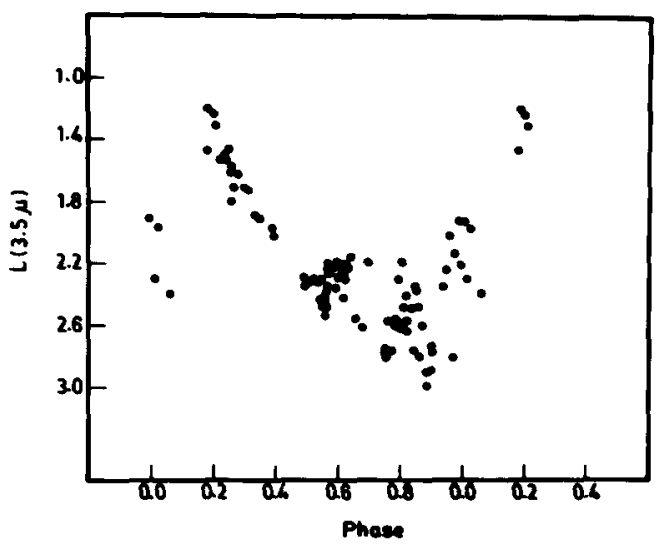

Fig. 1b. L magnitudes available during 1968-82 folded over a 1170 day period. 
Hence, we made a piece-wise analysis of the data which confirmed our suspicion. Further, we could find no single period which is present in all the different data segments. These results convincingly rule out the possibility that the observed irregular light fluctuations are caused by the superposition of several independent oscillations.

\section{LONG-TERM BEHAVIOUR}

\subsection{Visual light}

of the several periods present in the power spectrum, the most dominant is a long-term modulation with a period around 1200 day. In Fig. $1 \mathrm{a}$, we have $\mathrm{p}$ lotted the $\mathrm{V}$ and $\mathrm{B}-\mathrm{V}$ magnitudes of $\mathrm{R}$ CrB obtained near maximum against the photometric phases calculated using the following ephemeris:

$$
J D=2444294.0+1170.0 \mathrm{E}
$$

The initial epoch corresponds to the time of maximum brightness in $V$ for the 1170 day modulation. Both the period and the initial epoch are derived using least square method assuming a sinusoidal form for the variation. The total amplitude in $V$ is found to be around $0.15 \mathrm{mag}$. Apparent ly, $(B-V)$ does not show significant varia tion over this period. It is clear from Fig.la, that the shortterm fluctuations are superposed on this long-term behaviour.

\section{$3.2 \mathrm{~L}$ Band $(3.5 \mu)$ f lux}

The evidence of long-term modulation of the light draws one's attention towards the variability of infrared excess at $L$ band in $R \quad C r B$, noticed first by Humphreys and Ney (1974), and confirmed later by Strecker (1975) who derived a characteristic time of about 1100 days for the variation. The available $L$ band observations from 1968 to 1982 are plotted in Fig.1b with the above ephemeris. It is evident from the figure that the period fits the infrared data also fairly well. The variability in infrared excess is attributed to the pulsating circumste llar dust around $\mathrm{R} \mathrm{CrB}$ (Feast et a l. 1977). If true, this implies that there is some contribution to the optical variation from the circumste llar dust.

\section{RECENT RADIAL VELOCITY DATA}

In order to look for the suspected pulsation, $R$ CrB was observed spectroscopically on 27 nights during February-May 1985 with the $75-\mathrm{cm}$ Te lescope at Kavalur at a dispersion of $26 \mathrm{~A}^{\circ} \mathrm{mm}^{-1}$. In Fig.2a, we have plotted the radial ve locities derived by us against the corresponding Julian Days of observation. It is $\mathrm{clear}$ from the figure that the radial velocity of the star is variable, but no definite pattern for the variation could be seen. The total amplitude of variation is around $8 \mathrm{kms}^{-1}$ in about 50 days. We have obtained 


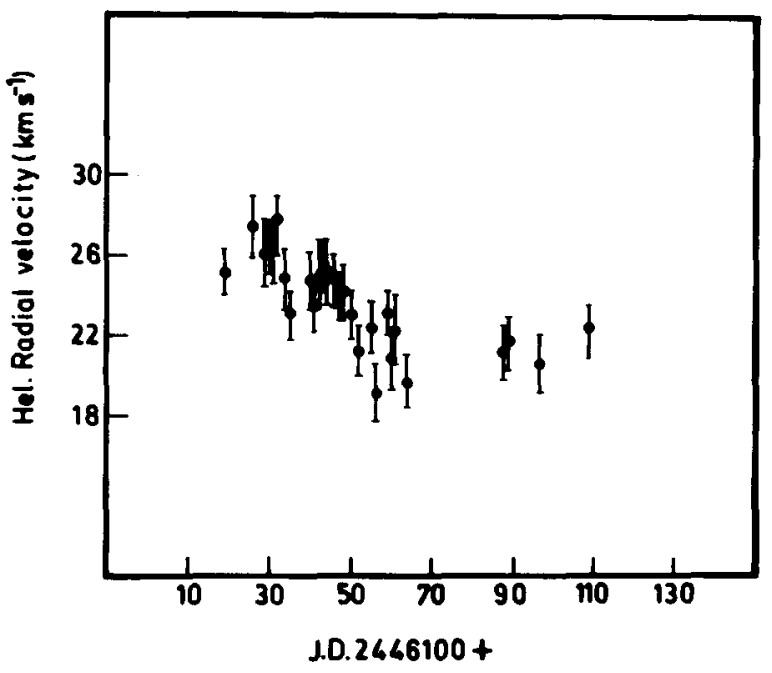

Fig. 2a. Radial velocities of $R \mathrm{CrB}$ obtained at Kavalur during February-March 1985.

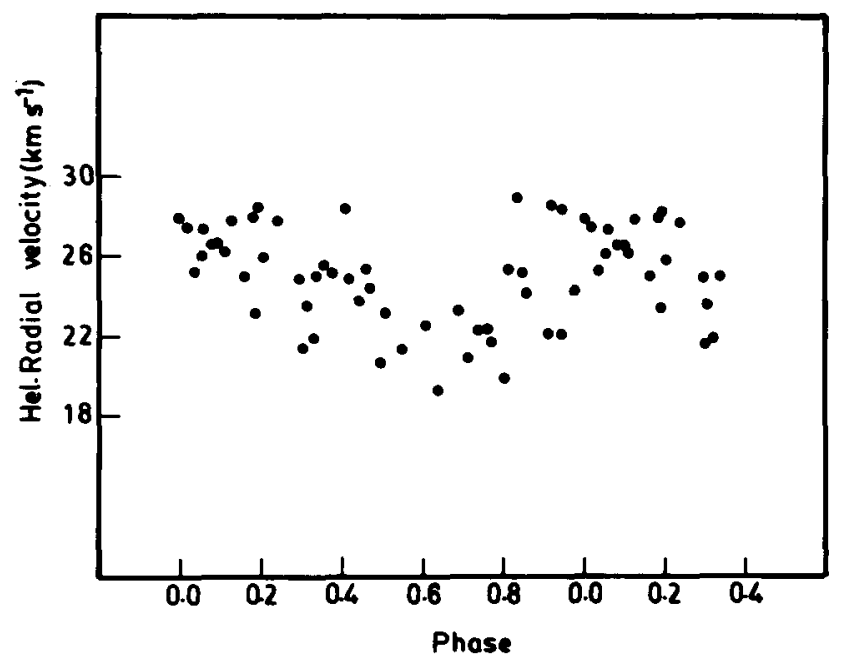

Fig. 2b. Radial velocities of $R \operatorname{Cr} B$ near maximum available since 1972 folded over a 47.18 day period. 
a few radial ve locity measurements during 1983 also. A period of 47.18 day is found to fit fairly we 11 a 17 our observations a long with those given by Fernie et a 1. (1972), as shown in Fig.2b. However, the period is a poor presentation if observations ear lier to 1972 are also included. An extended version of this paper will be published e lsewhere.

\section{REFERENCES}

Deeming, T.J. 1975, Astrophys. Sp. Sci. 36, 137.

Feast, M.W., Catchpole, R.M., L loyd Evans, T., Robertson, B.S.C., Dean, J.F. \& Bywater, R.A. 1977, Mon. Not. R. astr. Soc., $\underline{178}, 415$.

Fernie, J.D., Sherwood, V. \& DuPuy, D.L. 1972, Astrophys. J. 172, 383.

Humphreys, R.M. \& Ney, E.P. 1974, Astrophys. J. 190, 339.

Strecker, D.W. 1975, Astr. J. 80, 451. 


\section{DISCUSSION}

FEAST: Two points. One: there is some rough time timescale for the $L$ mag. I think it is unlikely that the period given by strecker will hold. I mean, later observers don't seem to confirm that period. So I think that all one wanted to say is that there is a timescale almost that long. It is interesting if you tie it to that sort of timescale in the visual, presuming that is due to the absorption in the shell. There seems to be an indication in the case of $\mathrm{S}$ Aps, there are peiods where the $\mathrm{V}$ light is down for a period of some thousands of days when it is at maximum. The other point that we agree on is that the radial velocities show periods of the order of 47 or 48 days, derived from Griffin's data which I mentioned. What I did not mention was that $\mathrm{Dr}$. Balona has analyzed a whole lot of $\mathrm{Dr}$. Herbig's data but here we don't see any periods in these early observations. If there are'pulsations, then they seem to be of very low amplitude. 